\title{
Bioinformatics-based identification of key pathways and candidate genes for estrogen-induced intrahepatic cholestasis using DNA microarray analysis
}

\author{
DONG XIANG, YANJIAO XU, WENXI HE, JINYU YANG, CHENGLIANG ZHANG and DONG LIU \\ Department of Pharmacy, Tongji Hospital Affiliated with Tongji Medical College, \\ Huazhong University of Science and Technology, Wuhan, Hubei 430030, P.R. China
}

Received December 22, 2018; Accepted April 12, 2019

DOI: $10.3892 / \mathrm{mmr} .2019 .10256$

\begin{abstract}
Estrogen-induced intrahepatic cholestasis (EIC) has increased incidence during pregnancy, and within women taking oral contraception and postmenopausal hormone replacement therapy. However, the pathology underlying EIC is not well understood. The aim of the present study was to identify key pathways and candidate genes in estrogen-induced intrahepatic cholestasis (EIC) that may be potential targets for diagnosis and treatment. A whole-genome microarray $(4 \mathrm{x} 44 \mathrm{~K})$ analysis of a $17 \alpha$-ethinylestradiol (EE)-induced EIC rat liver model was performed. Bioinformatics-based methods were used to identify key pathways and candidate genes associated with EIC. The candidate genes were validated using a reverse transcription quantitative polymerase chain reaction assay. A total of 455 genes were differentially expressed $(\mathrm{P}<0.05$ and fold change $>2.0$ ) following EE treatment, including 225 downregulated genes and 230 upregulated genes. Sulfotransferase family $1 \mathrm{E}$ member 1 , cytochrome P450 family 3 subfamily A member 2, carbonic anhydrase 3, leukotriene C4 synthase and ADAM metallopeptidase domain 8 were the 5 candidate genes identified to be differentially expressed and involved in the metabolism of estrogens and bile acids and the regulation of inflammation and oxidative stress. The Analyses of Gene Ontology enrichment, Kyoto Encyclopedia of Genes and Genomes pathways and protein-protein interaction network associated-modules identified several key pathways involved in the homeostasis of lipids and bile acids and in AMPK, p53 and Wnt signaling. These key pathways and candidate genes may have critical roles in the pathogenesis of EIC. In
\end{abstract}

Correspondence to: Professor Dong Liu or Professor Chengliang Zhang, Department of Pharmacy, Tongji Hospital Affiliated with Tongji Medical College, Huazhong University of Science and Technology, Wuhan, Hubei 430030, P.R. China

E-mail:1d2069@outlook.com

E-mail:ph3719@aliyun.com

Key words: intrahepatic cholestasis, DNA microarray, estrogen, pathway, candidate gene addition, reversing the abnormal expression of candidate genes or restoring the dysfunction of key pathways may provide therapeutic opportunities for patients with EIC.

\section{Introduction}

Intrahepatic cholestasis leads to systemic and intrahepatic retention of toxic bile acids that initially cause liver injury and subsequently result in biliary fibrosis and cirrhosis (1). Estrogens and their metabolites are well-known causes of estrogen-induced intrahepatic cholestasis (EIC) during pregnancy and in women taking oral contraception and postmenopausal hormone replacement therapy (2). Its pathogenesis arises primarily through genetic predisposition but also to a lesser extent by environment and immunology factors (3). Intrahepatic cholestasis of pregnancy (ICP) is the most common type of EIC, with an estimated prevalence of 0.4-1.5\% worldwide (4). Delayed diagnosis of ICP may increase the risks of stillbirth and early delivery (5). However, the pathogenic and physiopathological mechanisms of EIC are not well understood. EIC is currently treated with ursodeoxycholic acid (UDCA), but $30-50 \%$ of patients do not respond to UDCA treatment (6). Therefore, identification of key pathways and candidate genes for EIC may provide a greater understanding of this disease and aid in the development of novel drug targets.

Estrogen levels increase significantly during pregnancy and following administration of oral contraception and postmenopausal replacement therapy, particularly in patients with EIC (7). High estrogen levels induce acute cholestasis by impairing both bile flow and bile acid metabolism, causing downstream dysfunction of bile acid homeostasis (8). Accordingly, toxic bile acids accumulate in the liver, inducing oxidative stress and inflammatory reactions and the onset of liver injury (9). Clinical studies have indicated that oxidative stress responses are amplified in ICP patients compared to healthy pregnant women $(10,11)$. In addition, pro-inflammatory cytokines, including interleukin (IL)-6 and IL-17, are markedly increased in patients with ICP compared with healthy pregnant women $(12,13)$. Animal models of EIC have also demonstrated high expression levels of pro-inflammatory cytokines and an increased oxidative stress response $(14,15)$. 
The development of bioinformatics has provided new insights into the diagnosis and treatment of cholestatic liver diseases (16-18). Using integral microRNA (miRNA) and mRNA microarrays, Nakagawa et al (16) identified that the miR-425-mediated $\mathrm{T}$ cell receptor signaling pathway had an important role in regulating the synthesis of inflammatory cytokines in $\mathrm{CD} 4^{+} \mathrm{T}$ cells of patients with primary biliary cholangitis (PBC). Suppression of the T cell receptor signaling-associated regulator or miR-425 has therefore been suggested to be a promising immunotherapeutic strategy against PBC (16). Wang et al (17) identified serpin family $\mathrm{E}$ member $1(P A I-1)$ as the key gene in mice with extrahepatic cholestasis following bile duct ligation, and demonstrated that inhibiting PAI-1 may attenuate cholestatic liver diseases. These studies suggest that bioinformatics is a useful strategy to identify key pathways and candidate genes involved in disease as a foundation to develop new therapies. However, to the best of our knowledge, a comprehensive bioinformatical analyses of the cellular and molecular mechanisms underlying EIC has not been performed.

Cholestasis induced by $17 \alpha$-ethinylestradiol (EE) is a rodent model widely used to investigate the molecular mechanisms of EIC (19). The present study aimed to identify key pathways and candidate genes using a whole-genome microarray $(4 \mathrm{x} 44 \mathrm{~K})$ to analyze an EE-induced cholestatic rat model. The most significant differentially expressed genes (DEGs) involved in the metabolism of estrogen and bile acids and the regulation of inflammation and oxidative stress were shortlisted as candidate genes. In addition, Gene Ontology (GO), Kyoto Encyclopedia of Genes and Genomes (KEGG) pathway enrichment and protein-protein interaction (PPI) network analyses were performed to identify key pathways involved in EIC. A total of 5 candidate genes and several key pathways were identified, which may be candidate diagnostic biomarkers or therapeutic targets for EIC.

\section{Materials and methods}

Animals and treatments. A total of 12 male Sprague Dawley rats weighing $200 \pm 20 \mathrm{~g}$ were obtained from the Center of Experimental Animals of Hubei Province. All animals acclimated to the laboratory conditions for 1 week prior to the experiment and were kept at $25 \pm 2^{\circ} \mathrm{C}$ with a $12: 12 \mathrm{~h}$ light: dark cycle. Animals had ad libitum access to standard laboratory chow and tap water. The present study was approved by the Ethical Committee on Animal Experimentation of Tongji Medical College, Huazhong University of Science and Technology (Wuhan, China). Animals were randomly divided into control and model groups ( $\mathrm{n}=6$ per group). Control and model rats were administered subcutaneous (s.c.) propylene glycol (solvent of EE, $0.25 \mathrm{ml} / 100 \mathrm{~g}$, s.c.) or EE $(5 \mathrm{mg} / \mathrm{kg}$, s.c.) for 5 consecutive days, respectively. Rat conditions and body weight were recorded every day. Rats were fasted overnight following the last treatment prior to sacrifice. Bile flow was measured according to a previously described method (14). Blood samples and liver tissues were collected for subsequent analysis.

Biochemical determinations and histological analyses. Serum aspartate aminotransferase (ALT), alanine aminotransferase
(AST), alkaline phosphatase (ALP) and total bile acids (TBA) were analyzed using commercial kits (Nanjing Jiancheng Bioengineering Institute) according to the manufacturer's protocol. A section of the same liver parts from each rat was dissected and fixed in $10 \%$ formalin for $24 \mathrm{~h}$ in a room temperature, washed, dehydrated in alcohol gradients $(100,100,95,80$ and $75 \%, 2$ min each) and paraffin-embedded. Each slide was subsequently stained with hematoxylin $(0.2 \%, 5 \mathrm{~min}$, room temperature) and eosin $(0.5 \%, 30 \mathrm{sec}$, room temperature), and then assessed histologically with a light microscope (magnification, x200, EVOS FL Auto; Thermo Fisher Scientific, Inc.).

Gene expression analyses. Total RNA of 3 randomly selected rat liver tissues were extracted using RNAiso Plus (Takara Bio, Inc.) and purified using an RNeasy mini kit (Qiagen $\mathrm{GmbH}$ ) following the manufacturer's instructions. Total RNA was amplified and labeled with an Agilent Quick Amp Labeling kit (Agilent Technologies, Inc.). Each slide containing rat liver total RNA was hybridized with an Agilent Whole Rat Genome Oligo Microarray $(4 \mathrm{x} 44 \mathrm{~K})$ for $17 \mathrm{~h}$. Slides were then washed and scanned using an Agilent DNA Microarray Scanner (Agilent Technologies, Inc.). Data was extracted with Agilent Feature Extraction software v10.7 (Agilent Technologies, Inc.) and subsequently filtered for detection of normalization and significant changes, using $\mathrm{P}<0.05$ as the cut-off value, and DEGs compared to the control rats, using fold change $>2$ or $<0.5$ as the cut-off. The heatmap of DEGs was analyzed by SBC analysis system (Shanghai Biotechnology Corporation). Technical support was provided by the Shanghai Biotechnology Corporation.

Reverse transcription quantitative polymerase chain reaction $(R T-q P C R)$. Total RNA of all rat liver samples was isolated using TRIzol ${ }^{\circledR}$ reagent (Thermo Fisher Scientific, Inc.) and then reverse transcribed into cDNA using the PrimeScript ${ }^{\mathrm{TM}}$ RT Master Mix (Takara Biotechnology Co., Ltd.). The RT steps were: $37^{\circ} \mathrm{C}, 15 \mathrm{~min}$ and $85^{\circ} \mathrm{C}, 5 \mathrm{sec}$. Each cDNA was used as a template for RT-qPCR amplification, performed using SYBR Premix Ex Taq ${ }^{\mathrm{TM}}$ (Takara Biotechnology Co., Ltd.) and pairs of forward/reverse primers for the candidate genes and rat $\beta$-actin gene. The qPCR steps were: initial denaturation, $95^{\circ} \mathrm{C}, 30 \mathrm{sec}$, PCR reaction, $95^{\circ} \mathrm{C}, 5 \mathrm{sec}, 60^{\circ} \mathrm{C}$, $34 \mathrm{sec}, 40$ cycles. Independent reactions were performed in triplicate using an Applied Biosystems StepOnePlus Real-time PCR system (Applied Biosystems). Threshold cycle (Cq) values were normalized to those for $\beta$-actin $(\Delta \mathrm{Cq})$. The $2^{-\Delta \Delta \mathrm{Cq}}$ method was used to calculate the relative changes of gene expression (20). The primer sequences of candidate genes and $\beta$-actin are presented in Table I.

Functional enrichment analyses of the DEGs. Functional annotation of DEGs was performed using the online Database for Annotation, Visualization, and Integrated Discovery (DAVID; https://david.ncifcrf.gov/). DAVID provides comprehensive functional annotations tools for the analysis of the genes and proteins included in the database, for users to obtain biological information. The functions of the DEGs was determined via GO terms and KEGG pathway enrichment using DAVID, with $\mathrm{P}<0.05$ used as the cut-off for statistical significance. 
Table I. Sequence of primers for 6 candidate genes and $\beta$-actin for reverse transcription quantitative polymerase chain reaction.

\begin{tabular}{|c|c|c|}
\hline Gene name & Direction & Sequence $\left(5^{\prime}-3^{\prime}\right)$ \\
\hline \multirow[t]{2}{*}{ Sult1e1 } & Forward & ATTCAATGCTGCCAGAGACC \\
\hline & Reverse & TCATTTGCTGCTGGTAGTGC \\
\hline \multirow[t]{2}{*}{ Сур3а2 } & Forward & TGACTGCTCTTGATGCATGGTT \\
\hline & Reverse & ATCACAGACCTTGCCAACTCCT \\
\hline \multirow[t]{2}{*}{ Car3 } & Forward & GCTCCTTTTAATCACTTCGACC \\
\hline & Reverse & AGCCACACAATGCACTCCTC \\
\hline \multirow[t]{2}{*}{ Ltc4s } & Forward & GAAGAACTTTCCACGTGTCG \\
\hline & Reverse & GTGCAGCCATTGCCACTAGC \\
\hline \multirow[t]{2}{*}{ Adam8 } & Forward & GCCTCGGACCTTAGAAAT \\
\hline & Reverse & GGCATAACGGCTGATGTA \\
\hline \multirow[t]{2}{*}{$\beta$-actin } & Forward & CACCCGCGAGTACAACCTTC \\
\hline & Reverse & СССАТАСССАССАТСАСАСС \\
\hline
\end{tabular}

Sult1e1, sulfotransferase family 1E member 1; Cyp3a2, cytochrome P450 family 3 subfamily A member 2; Car3, carbonic anhydrase 3; Ltc4s, leukotriene C4 synthase; Adam8, ADAM metallopeptidase domain 8 .

Construction of PPI networks and analysis of modules. The Search Tool for Retrieval of Interacting Genes and Proteins (STRING) database (v10.0; http://string-db.org/) was used for PPI network construction of the DEGs. Genes without associations with other genes were removed. Interactions with a combined score $>0.4$ were considered significant. PPI networks were used for module screening using the Molecular Complex Detection (MCODE) in Cytoscape (v3.6.0) bioinformatics integration platform (21). Significant modules were those with scores $>3$ and nodes $>4$. KEGG pathway enrichment analysis for DEGs was performed in each module using ClueGO software (version 2.5.0, Institute for Genomics and Bioinformatics Graz University of Technology, Graz, Austria).

Statistical analyses. Statistical analyses were performed using SPSS software v13.0 (SPSS Software, Inc.). All data are presented as the mean \pm standard deviation. A Student's t-test was used to compare the differences between two groups. $\mathrm{P}<0.05$ was considered to indicate a statistically significant difference.

\section{Results}

EE-induced cholestasis and liver injury. The body weight of the control rats gradually increased, while that of model rats initially increased, but then fell. A significant difference $(\mathrm{P}<0.01)$ in body weight between control and model rats was observed at days 5 and 6 (Fig. 1A). Bile flow was suppressed $(\mathrm{P}<0.01)$ in the EE-treated rats (Fig. 1B). Serum ALT, AST, ALP and TBA levels were significantly increased in the EE-induced rats compared with the control rats (Fig. 1C). Histological evaluation of the hepatic tissues indicated that control rats exhibited normal structures, while there was evidence of neutrophil infiltration, edema and hepatic necrosis
Table II. Top 5 downregulated and upregulated differentially expressed genes $(\mathrm{n}=3)$.

\begin{tabular}{llcc}
\hline Direction & \multicolumn{1}{c}{ Symbol } & $\log _{10} \mathrm{FC}$ & P-values \\
\hline Downregulated & Car3 & -1.988 & 0.0019 \\
& Sult1e1 & -1.966 & 0.0272 \\
& Cyp3a2 & -1.531 & 0.0322 \\
& LOC100912610 & -1.444 & 0.0277 \\
Upregulated & Ltc4s & -1.396 & 0.0042 \\
& Rasd2 & 1.894 & 0.0034 \\
& Adam8 & 1.882 & 0.0074 \\
& Pvalb & 1.859 & 0.0153 \\
& Slc5a1 & 1.705 & 0.0010 \\
& Cited4 & 1.638 & 0.0009 \\
\hline
\end{tabular}

DEGs, differentially expressed genes; FC, fold change; Sult1e1, sulfotransferase family $1 \mathrm{E}$ member 1 ; Cyp3a2, cytochrome P450 family 3 subfamily A member 2; Car3, carbonic anhydrase 3; Ltc4s, leukotriene C4 synthase; Rasd2, RASD family member 2; Adam8, ADAM metallopeptidase domain 8; Pvalb, paravalbumin; Slc5a1, solute carrier family 5 member 1; Cited4, Cbp?P300 interacting transactivator with Glu/Asp rich carboxy-terminal domain 4 .

in model rats (Fig. 1D). These results were in concordance with the previously published data (22) and thereby suggest that $\mathrm{EE}$ induced cholestasis and liver injury in rats.

Identification of DEGs and candidate genes. Following preprocessing of the microarray data, the gene expression data were normalized and visualized with a box plot. A good normalization performance was obtained (Fig. 2A). In total, 455 DEGs were identified, including 225 downregulated genes and 230 upregulated genes. The heatmap function was used to observe DEGs in different samples (Fig. 2B). The top 5 downregulated and upregulated DEGs are listed in Table II. Genes were thereafter shortlisted as candidate genes based on evidence of their involvement in the metabolism of estrogen and bile acids or in the regulation of inflammatory reaction and oxidative stress, either from the literature or the data from the present study. These were sulfotransferase family $1 \mathrm{E}$ member 1 (Sult1e1), cytochrome P450 family 3 subfamily A member 2 (Cyp3a2), carbonic anhydrase 3 (Car3), leukotriene C4 synthase (Ltc $4 s)$ and ADAM metallopeptidase domain 8 (Adam8). To confirm the reliability of DNA microarray data, the mRNA expression levels of these candidate genes were investigated by RT-qPCR. The results were consistent with those from the DNA microarray data (Fig. 2C).

Functional enrichment analysis for gene expression. To explore the functions of DEGs, GO and KEGG pathway enrichment analyses were performed using the DAVID. As demonstrated in Fig. 3, analysis of GO indicated that the upregulated genes were primarily enriched in the regulation of protein binding, calcium ion binding, epithelial cell differentiation and extracellular exosome, while the downregulated genes were primarily enriched in regulators of iron ion binding, heme binding, aromatase activity and oxidation-reduction. 

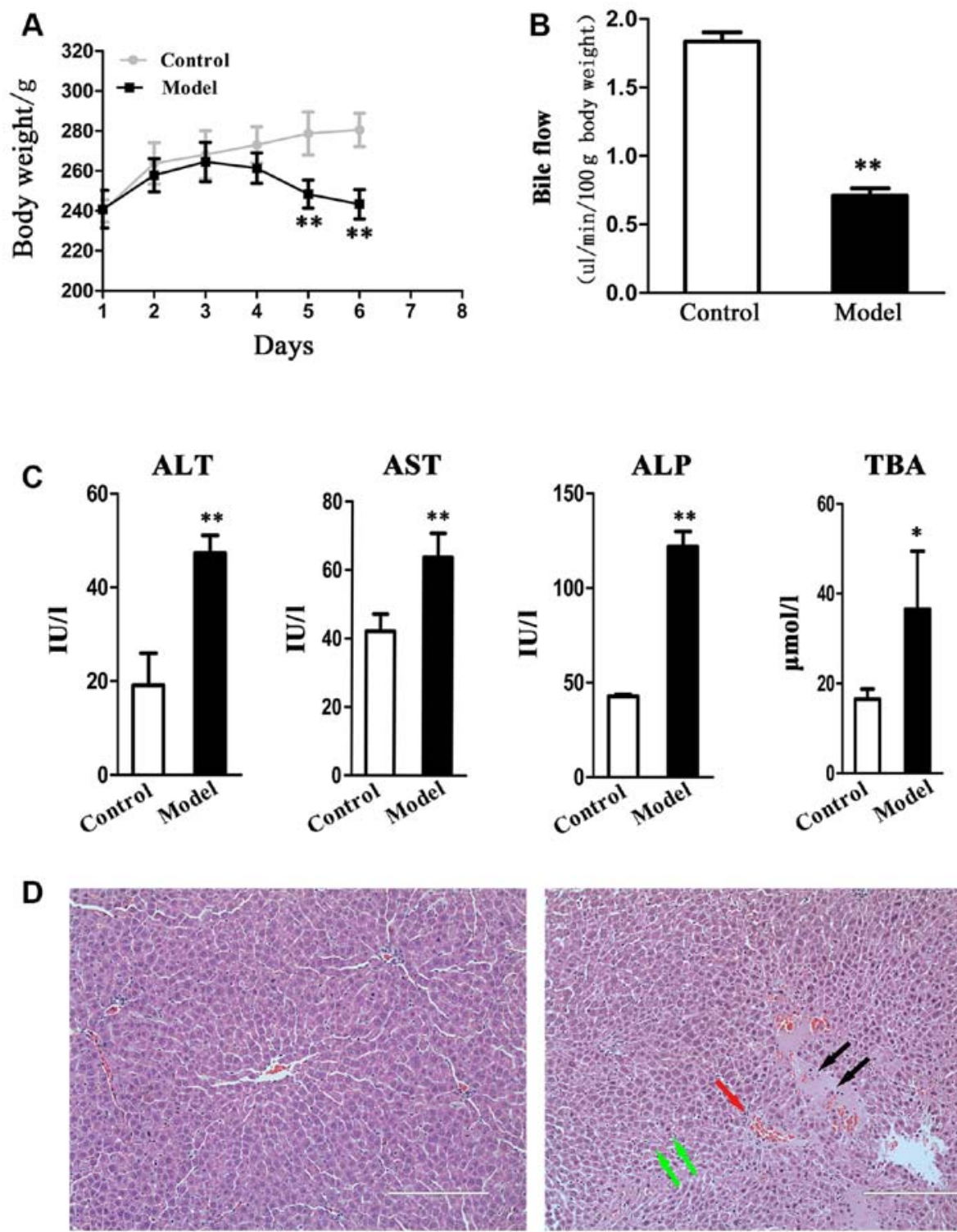

Control

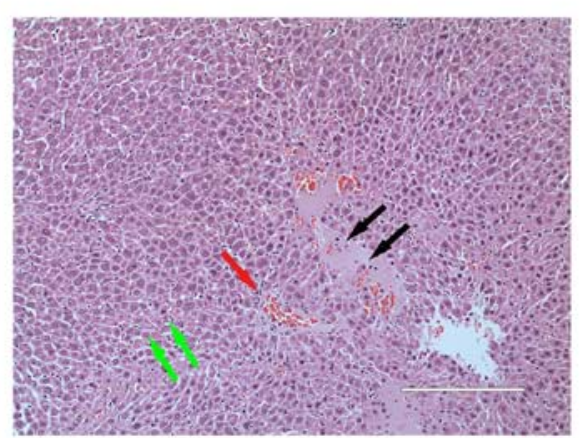

Model

Figure 1. 17 $\alpha$-ethinylestradiol-induced cholestasis and liver injury. (A) Changes in body weight and (B) bile flow in EIC rats. Bile was collected in a 10-minute period to monitor the flow. (C) Serum biochemical index of AST, ALT, ALP and TBA. (D) The images of representative hematoxylin and eosin stained liver sections (magnification, x200). Liver necrosis, neutrophil infiltration and edema are marked by black, red, and green arrows, respectively. Data are presented as the mean \pm standard deviation $(\mathrm{n}=6) .{ }^{*} \mathrm{P}<0.05$ and ${ }^{* *} \mathrm{P}<0.01$ vs. control. EIC, estrogen-induced intrahepatic cholestasis; AST, aspartate aminotransferase; ALT, alanine aminotransferase; ALP, alkaline phosphatase; TBA, total bile acids.

KEGG analysis was used for the identification of significantly enriched pathways of the DEGs. The upregulated DEGs were primarily enriched in the pathways of 5'AMP-activated protein kinase (AMPK) and prolactin and those involved in protein digestion, protein absorption and fatty acid metabolism. The downregulated DEGs were primarily enriched in steroid hormone biosynthesis, retinol metabolism, metabolic pathways and arachidonic acid metabolism. A total of 3 pathways also enriched in the downregulated DEGs were involved in regulating bile acid homeostasis, including bile secretion, peroxisome proliferator-activated receptor (PPAR) signaling and primary bile acid biosynthesis.

PPI network construction and module selection. Protein interactions of DEGs were predicted by the STRING database and then the PPI networks were constructed for those with a combined protein pair score $>0.4$ (Fig. 4). Modules of genes in the PPI network were identified by the MCODE plugin in Cytoscape. The top 4 significant modules were selected, and the cellular pathways of the genes involved in these modules were analyzed using ClueGO. As demonstrated in Fig. 5, the cellular pathways were involved in steroid hormone biosynthesis, retinol metabolism, arachidonic acid metabolism, bile secretion, and $\mathrm{p} 53$ and Wnt signaling.

\section{Discussion}

EIC is characterized by high estrogen levels, accumulation of toxic bile acids, hepatic inflammation and oxidative stress, which leads to the fibrosis, cirrhosis and eventually failure of the liver (23). Liver transplantation remains the only strategy for patients with the end-stage EIC (24). For EICs detected at 


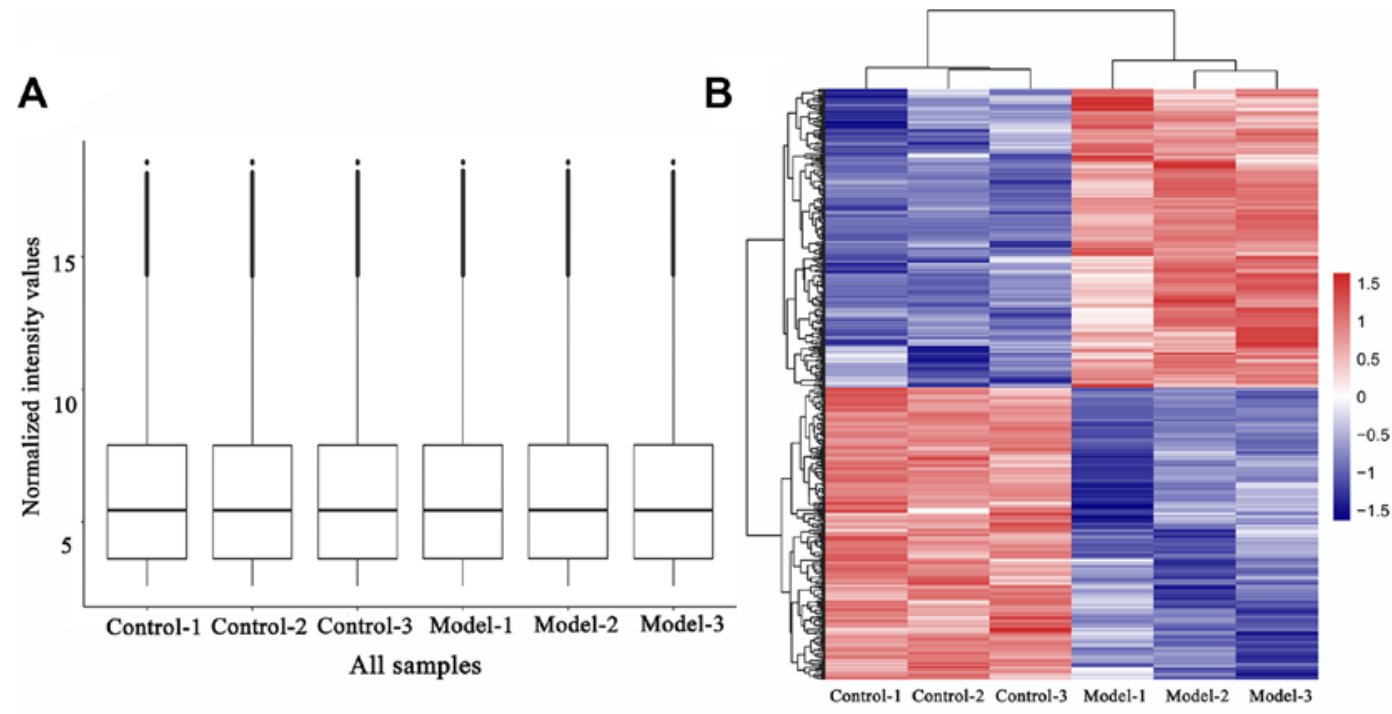

C

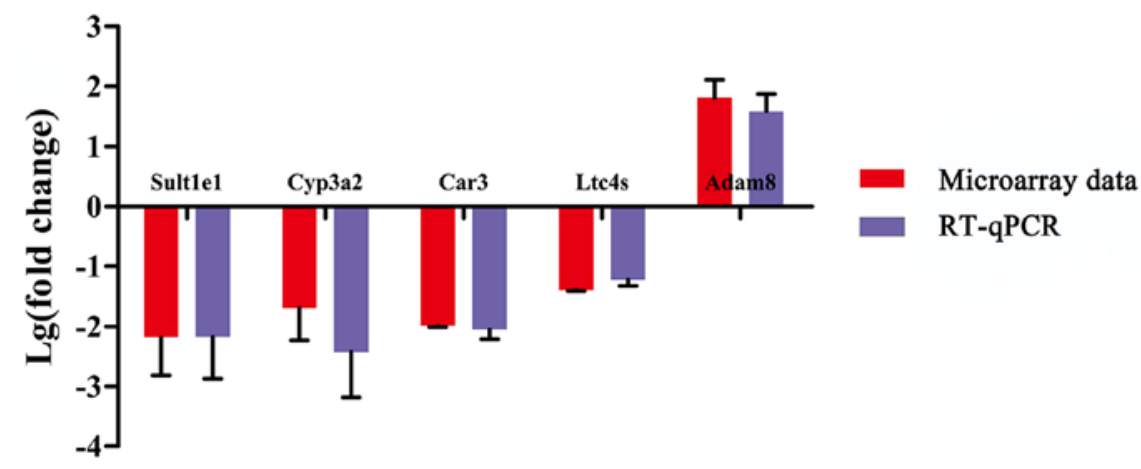

Figure 2. Cluster analysis of DEGs and a selection of candidate genes. (A) Box plot for normalized gene expression data. The medians are similar, indicating a high level of normalization. (B) Cluster analysis of DEGs is exhibited in a heat map $(n=3)$. Red indicates upregulated expression, while blue indicates downregulated expression. (C) RT-qPCR confirmation for the 5 candidate genes $(n=6)$. Each column represents the $\log _{10}$ fold change of candidate genes normalized to $\beta$-actin. The data are presented as the mean \pm standard deviation. DEGs, differentially expressed genes; RT-qPCR, reverse transcription quantitative polymerase chain reaction; Sult1e1, sulfotransferase family 1E member 1; Cyp3a2, cytochrome P450 family 3 subfamily A member 2; Car3, carbonic anhydrase 3; Ltc4s, leukotriene C4 synthase; Adam8, ADAM metallopeptidase domain 8.

earlier stages, UDCA and obeticholic acid, a nuclear receptor agonist, are the first- and second-line drugs; however, their curative effects are limited (25). In addition, the mechanisms involved in the pathogenesis and physiopathology of EIC are not well understood. Understanding the molecular mechanisms involved in the development and progression of EIC is therefore crucial for developing and evaluating diagnostic and management strategies.

In the present study, a whole-genome microarray was used to identify key pathways and candidate genes within EE-induced cholestatic rats. The results identified a total of 455 DEGs, including 225 downregulated genes and 230 upregulated genes. Among the top upregulated and downregulated genes, those involved in the metabolism of estrogens and bile acids, and the regulation of inflammation and oxidative stress, were identified and validated as candidate genes. These were Sult1e1, Cyp3a2, Car3, Ltc4s and Adam8. The elevated estrogen levels that are typically observed in patients with EIC and animal models induce dysfunction of bile acid hemostasis through activating estrogen-responsive receptors, leading to the accumulation of toxic bile acids in the liver and the development of liver injury (1). A phase II drug-metabolizing enzyme, Sult1e1, is known to catalyze the sulfoconjugation of estrogens and their metabolites (26). It has been demonstrated Sult1e1 is downregulated during several liver diseases, including cholestasis, and this is associated with elevated serum estrogen $(27,28)$. An additional study investigating livers in a cystic fibrosis mouse model indicated that elevated hepatic Sult1e1 levels may result in decreased levels of estrogens and decreased expression of estrogen-responsive receptors (29). Cyp3a2, a human ortholog of cytochrome P450 family 3 subfamily A member 4 (CYP3A4), is a phase I detoxifying cytochrome P450 enzyme that catalyzes the elimination of bile acid (30) and thereby decreases toxic bile acid levels in the liver (31). Numerous studies have demonstrated that approved drugs including rifampicin, phenobarbital and bezafibrate protect against cholestasis through inducing CYP3A4 activity and detoxifying bile acid levels (32-34). In the present study, the extremely low expression of Sult1e1 and Cyp3a2 may limit the metabolism of estrogens and bile acids and lead to their accumulation in the liver. Therefore, reversing the expression of Sultlel and Cyp3a2 may be a target for EIC therapy. 
A

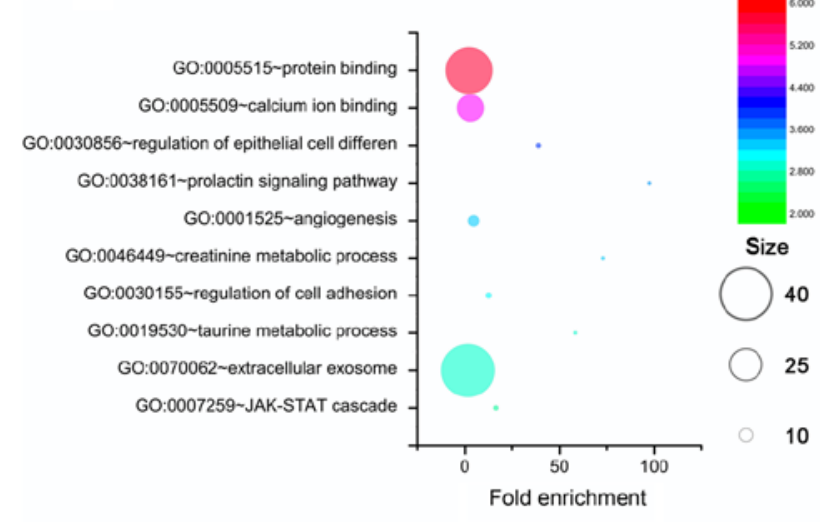

C

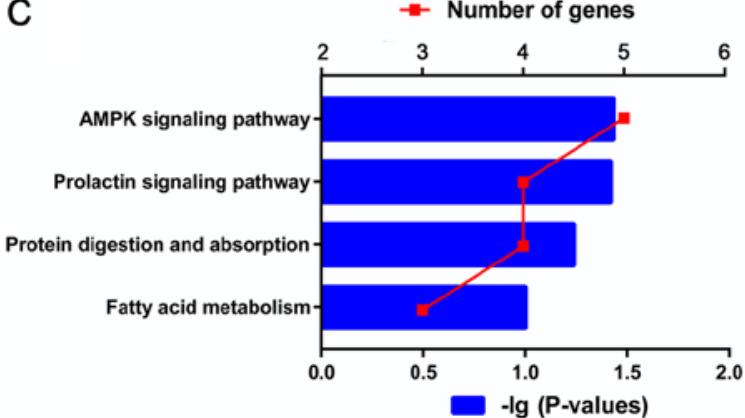

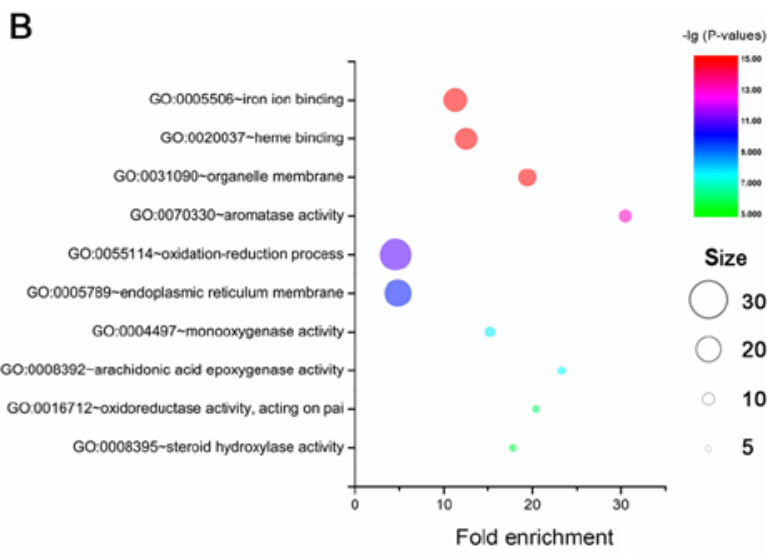

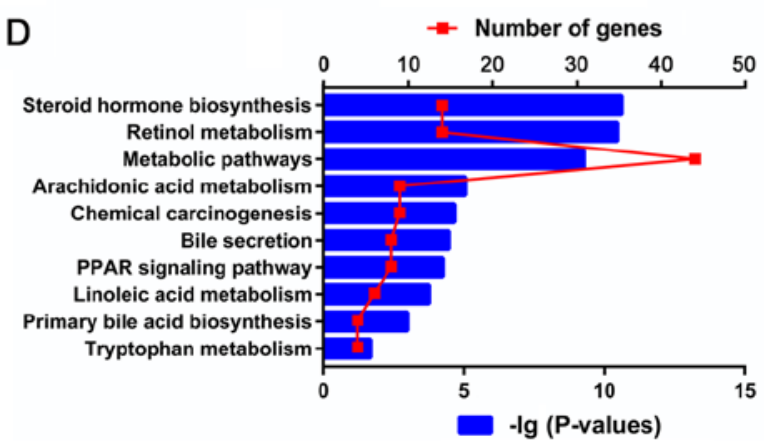

Figure 3. Functional enrichment analysis of DEGs. GO enrichment analysis of (A) upregulated DEGs and (B) downregulated DEGs. GO analysis classified into 3 groups, including 'Biological Process', 'Cellular Component' and 'Molecular Function'. KEGG enrichment analysis of (C) upregulated DEGs and (D) downregulated DEGs. DEGs, differentially expressed genes; GO, Gene ontology; KEGG, Kyoto Encyclopedia of Genes and Genomes; AMPK, 5'AMP-activated protein kinase.

Accumulation of hepatic bile acids often results in oxidative stress and inflammation, which then leads to hepatocellular injury (9). Car3, a human ortholog of $C A 3$, is responsible for catalyzing the hydration of carbon dioxide in response to oxidative stress, but is nonetheless expressed at low levels in EIC. It has been demonstrated that Car3 serves an important role in glutathionylation during cellular oxidative stress (35). Compared with parental cells, Car3-transfected cells exhibited lower levels of the intracellular reactive oxygen species (36). More importantly, Car3 decreases the toxicity of hydrophobic bile acids on biliary epithelium in bicarbonate-rich hydrocholeresis (37). Additionally, Car3 is involved in mediating oxidative stress in various tissues and pathological processes, including in skeletal muscle and during alcoholic liver disease and aging (38-40). As a nuclear-membrane enzyme, Ltc 4 s catalyzes the conjugation of leukotriene A4 to glutathione, generating leukotriene $\mathrm{C} 4$, which is the first reaction in the synthesis of cysteinyl leukotrienes (LTs) (41). The previously described role of LTs strongly implicates them in the pathogenesis of inflammatory diseases (41-42). In addition, increasing evidence has demonstrated that LTs are associated with cholestasis, hepatic inflammation during metabolic disease and fulminant hepatic failure (42). Adam8 is a member of the ADAM protein family, which serves critical roles in certain liver diseases. Li et al (43), for example, demonstrated that Adam8 promoted liver injury by inhibiting the proliferation of hepatocytes and promoting angiogenesis, and by affecting the metabolic function of the liver during acute liver injury induced by $\mathrm{CCl}_{4}$. Accordingly, neutralization of Adam8 ameliorated liver injury and accelerated liver repair. Concomitantly, Higuchi et al (44) identified that oxazolone-induced contact hypersensitivity reactions were more severe in Adam8 transgenic mice compared with wild-type mice. Adam8, directly or indirectly, regulates leukocyte infiltration in mice (44). The microarray data and RT-qPCR results from the present study indicated that Car3, Ltc $4 s$ and Adam 8 were the most significantly altered DEGs in the EE-induced rat model. Therefore, these 3 candidate genes may have crucial roles in the regulation of oxidative stress and inflammation during the pathogenesis of EIC.

Key pathways underlying EIC were investigated through KEGG and PPI network-associated module analyses in the present study. The KEGG analysis indicated that the AMPK signaling pathway was primarily enriched in the upregulated DEGs. It has previously been demonstrated that the activation of AMPK signaling is integral to the EE-mediated disruption of the expression of bile acid transporters and promoting the EIC process (45). In addition, inhibition of AMPK activation attenuated EE-induced cholestasis in vitro and in vivo (45). AMPK signaling is also crucial for the pathogenesis of cholestatic liver injury involved in regulating hepatic polarity, inflammation and fibrosis (46). Fatty acid metabolism was enriched in the upregulated DEGs, whereas steroid hormone biosynthesis and PPAR signaling pathway were enriched in the downregulated DEGs. These 3 pathways are all involved in lipid metabolism. It has been demonstrated previously that alterations in lipid metabolism may promote inflammation, fibrosis and proliferation in a mouse model of chronic cholestatic liver injury (47). Fenofibrate is a PPAR $\alpha$ agonist that protects against EE-induced 


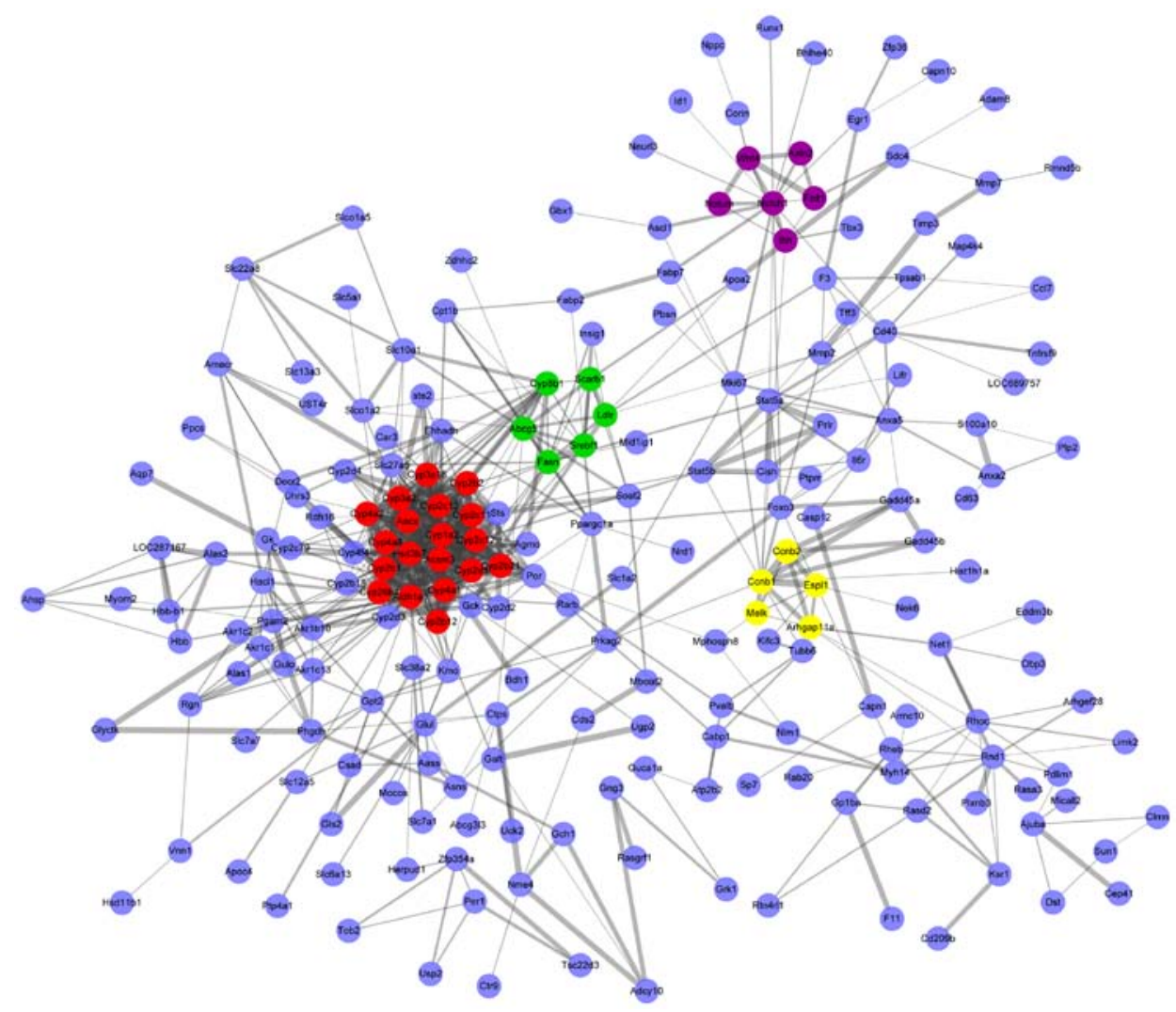

Figure 4. PPI network analysis of differentially expressed genes. The PPI network was constructed with the combined score of protein pairs $>0.4$. Line thickness indicates the strength of the association. The top 4 significant modules were selected according to the Molecular Complex Detection. The red dots represent module 1, the green dots represent module 2, the yellow dots represent module 3 and the purple dots represent module 4. PPI, protein-protein interaction.

A

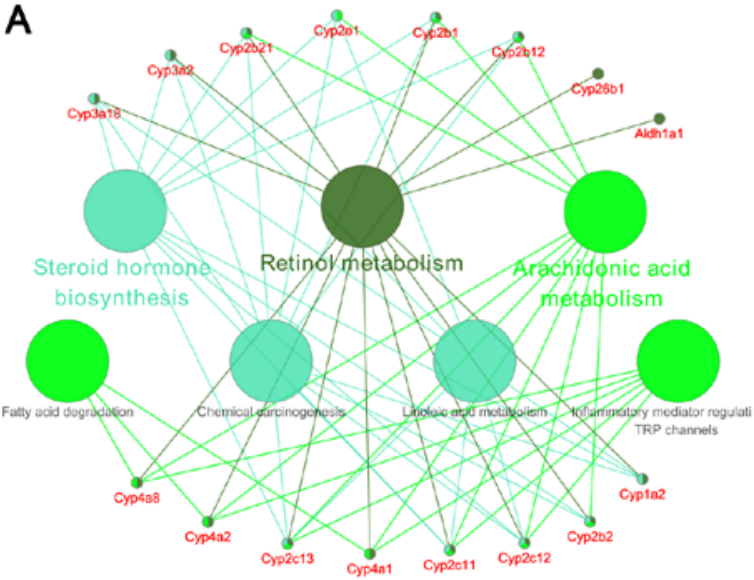

B

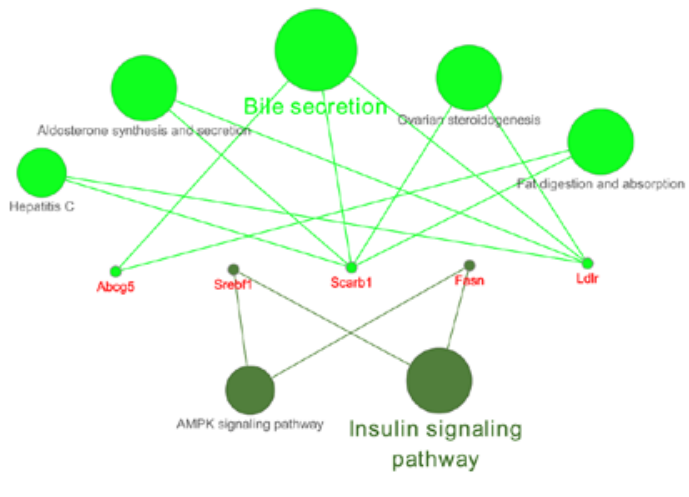

C

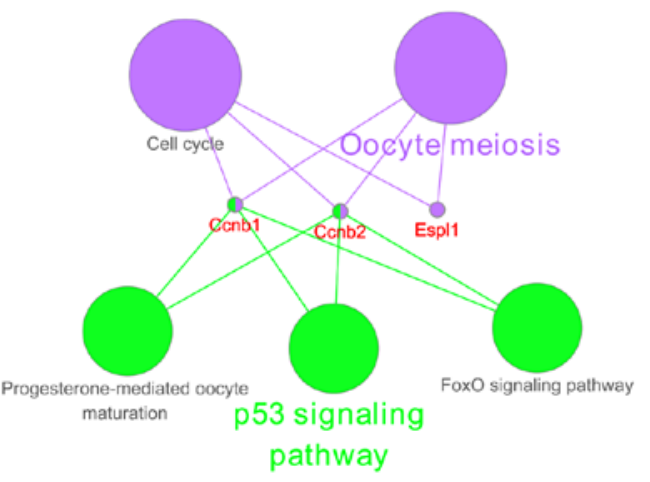

D

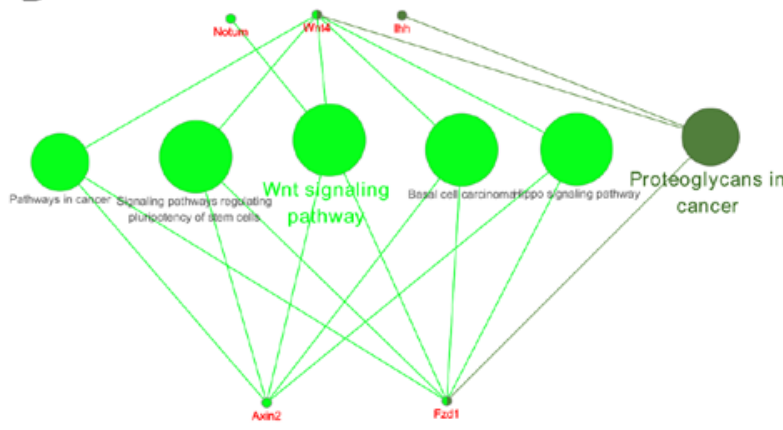

Figure 5. Pathway enrichment analysis of the top 4 modules from the protein-protein network by using ClueGO software. (A) Module 1. (B) Module 2. (C) Module 3. (D) Module 4. 
cholestasis in mice (48). In addition, it is well recognized that bile acid accumulation contributes to cholestasis. The results of the present study indicated that bile secretion and primary bile acid biosynthesis were primarily enriched in the downregulated DEGs, which suggests that bile acid homeostasis is disrupted during EE-induced cholestasis. In previous decades, numerous studies have struggled to regulate the expression of bile acid transporters, metabolic enzymes and nuclear receptors to improve bile acid homeostasis in cholestatic liver diseases (49). Accordingly, the inhibition of AMPK signaling or maintenance of lipid and bile acid homeostasis may provide potential strategies for EIC treatment.

The p53 and Wnt signaling pathways were identified as key pathways based on the data from the PPI network associated-modules. The role of p53 signaling on cholestasis is controversial. Chen et al (50) identified that the p53 activator doxorubicin attenuated cholic acid-induced cholestasis in mice through promoting the deposition of bile acid and alleviation of cholestatic syndrome, and that cholic acid-induced cholestatic liver injury was aggravated in p53-knockout mice. Concomitantly, other studies hypothesized that p53 signaling served an important role in promoting apoptosis during cholestatic liver injury $(51,52)$. Future studies are required to determine the role of p53 signaling on EIC and other cholestatic diseases. In addition, it has been demonstrated that Wnt signaling is a critical regulator of the pathophysiology of cholestasis $(53,54)$. Wnt signaling regulates hepatobiliary repair in cholestatic mice (54) and controls intrahepatic biliary network formation in zebrafish (53). Therefore, the regulation of p53 and Wnt signaling pathways may also provide novel insights into EIC treatment.

There are several limitations of the present study. Due to the fact that there are limited data available on the pathways and genes involved in EIC, the identified key pathways and candidate genes were not discussed in detail. Additionally, these results were generated from rat liver samples, which may cause tissue or sample heterogeneity in independent studies. Finally, only bioinformatics-based evidence and part of RT-qPCR data were presented, and additional studies are therefore required to confirm the functions of these candidate genes and key pathways in EIC, and to verify the results.

In summary, the present study identified 5 candidate genes and several key pathways that were demonstrated to be important in the pathogenesis of EIC. These pathways were involved in the homeostasis of lipids and bile acids and in AMPK, p53 and Wnt signaling. The results of the present study indicated that these candidate genes and key pathways may serve critical roles in the development and progression of EIC. Reversing the abnormal expression of candidate genes and dysfunction of key pathways may provide opportunities for novel EIC therapies.

\section{Acknowledgements}

Not applicable.

\section{Funding}

The present study was supported by grants from National Natural Science Foundation of China (grant nos. 81670521 and 81803798).

\section{Availability of data and materials}

The microarray data and other data sets in the present study are available from the corresponding author on reasonable request.

\section{Authors' contributions}

DL and CZ designed, supervised and revised the study. DX and $\mathrm{CZ}$ collected and analyzed the data, and wrote the manuscript. YX and WH contributed to microarray data collection and analysis. JY assisted in performing experiments and analysis of the data. All authors reviewed and approved the manuscript.

\section{Ethics approval and consent to participate}

The present study was approved by the Ethical Committee on Animal Experimentation of Tongji Medical College, Huazhong University of Science and Technology (Wuhan, China).

\section{Patient consent for publication}

Not applicable.

\section{Competing interests}

The authors declare that they have no competing interests.

\section{References}

1. Marrone J, Soria LR, Danielli M, Lehmann GL, Larocca MC and Marinelli RA: Hepatic gene transfer of human aquaporin-1 improves bile salt secretory failure in rats with estrogen-induced cholestasis. Hepatology 64: 535-548, 2016.

2. Schreiber AJ and Simon FR: Estrogen-induced cholestasis: Clues to pathogenesis and treatment. Hepatology 3: 607-613, 1983.

3. Stieger B, Fattinger K, Madon J, Kullak-Ublick GA and Meier PJ: Drug- and estrogen-induced cholestasis through inhibition of the hepatocellular bile salt export pump (Bsep) of rat liver. Gastroenterology 118: 422-430, 2000.

4. Hillman SC, Stokes-Lampard H and Kilby MD: Intrahepatic cholestasis of pregnancy. BMJ 353: i1236, 2016.

5. Kondrackiene $\mathbf{J}$ and Kupcinskas L: Intrahepatic cholestasis of pregnancy-current achievements and unsolved problems. World J Gastroenterol 14: 5781-5788, 2008.

6. Zhang Y, Lu L, Victor DW, Xin Y and Xuan S: Ursodeoxycholic acid and S-adenosylmethionine for the treatment of intrahepatic cholestasis of pregnancy: A meta-analysis. Hepat Mon 16: e38558, 2016.

7. Zucchetti AE, Barosso IR, Boaglio AC, Basiglio CL, Miszczuk G, Larocca MC, Ruiz ML, Davio CA, Roma MG, Crocenzi FA and Pozzi EJ: G-protein-coupled receptor 30/adenylyl cyclase/protein kinase A pathway is involved in estradiol 17ss-Dglucuronide-induced cholestasis. Hepatology 59: 1016-1029, 2014.

8. Reyes H and Simon FR: Intrahepatic cholestasis of pregnancy: An estrogen-related disease. Semin Liver Dis 13: 289-301, 1993.

9. Copple BL, Jaeschke H and Klaassen CD: Oxidative stress and the pathogenesis of cholestasis. Semin Liver Dis 30: 195-204, 2010.

10. Ozler A, Ucmak D, Evsen MS, Kaplan I, Elbey B, Arica M and Kaya M: Immune mechanisms and the role of oxidative stress in intrahepatic cholestasis of pregnancy. Cent Eur J Immunol 39: 198-202, 2014.

11. Sanhal CY, Daglar K, Kara O, Yilmaz ZV, Turkmen GG, Erel O, Uygur D and Yucel A: An alternative method for measuring oxidative stress in intrahepatic cholestasis of pregnancy: Thiol/disulphide homeostasis. J Matern Fetal Neonatal Med 31: 1477-1482, 2018.

12. Biberoglu E, Kirbas A, Daglar K, Kara O, Karabulut E, Yakut HI and Danisman N: Role of inflammation in intrahepatic cholestasis of pregnancy. J Obstet Gynaecol Res 42: 252-257, 2016. 
13. Kirbas A, Biberoglu E, Ersoy AO, Dikmen AU, Koca C, Erdinc S, Uygur D, Caglar T and Biberoglu K: The role of interleukin-17 in intrahepatic cholestasis of pregnancy. J Matern Fetal Neonatal Med 29: 977-981, 2016.

14. Liu D, Wu T, Zhang CL, Xu YJ, Chang MJ, Li XP and Cai HJ: Beneficial effect of Calculus Bovis Sativus on 17 $\alpha$-ethynylestradiolinduced cholestasis in the rat. Life Sci 113: 22-30, 2014.

15. Yamamoto Y, Moore R, Hess HA, Guo GL, Gonzalez FJ, Korach KS, Maronpot RR and Negishi M: Estrogen receptor alpha mediates 17alpha-ethynylestradiol causing hepatotoxicity. J Biol Chem 281: 16625-16631, 2006.

16. Nakagawa R, Muroyama R, Saeki C, Goto K, Kaise Y, Koike K, Nakano M, Matsubara Y, Takano K, Ito S, et al: miR-425 regulates inflammatory cytokine production in CD4(+) T cells via $\mathrm{N}$-Ras upregulation in primary biliary cholangitis. J Hepatol 66 : 1223-1230, 2017

17. Wang H, Vohra BP, Zhang Y and Heuckeroth RO: Transcriptional profiling after bile duct ligation identifies PAI-1 as a contributor to cholestatic injury in mice. Hepatology 42: 1099-1108, 2005

18. Sakamoto T, Morishita A, Nomura T, Tani J, Miyoshi H, Yoneyama $\mathrm{H}$, Iwama $\mathrm{H}$, Himoto $\mathrm{T}$ and Masaki T: Identification of microRNA profiles associated with refractory primary biliary cirrhosis. Mol Med Rep 14: 3350-3356, 2016.

19. Carreras FI, Lehmann GL, Ferri D, Tioni MF, Calamita G and Marinelli RA: Defective hepatocyte aquaporin- 8 expression and reduced canalicular membrane water permeability in estrogen-induced cholestasis. Am J Physiol Gastrointest Liver Physiol 292: G905-G912, 2007.

20. Crocenzi FA, Sánchez PE, Pellegrino JM,Favre CO, Rodríguez GE, Mottino AD, Coleman R and Roma MG: Beneficial effects of silymarin on estrogen-induced cholestasis in the rat: A study in vivo and in isolated hepatocyte couplets. Hepatology 34: 329-339, 2001

21. Livak JK and Schmittgen DT: Analysis of relative gene expression data using real-time quantitative PCR and the 2(-Delta Delta C(T)) method. Methods 25: 402-408, 2001.

22. Shannon P, Markiel A, Ozier O, Baliga NS, Wang JT, Ramage D, Amin N, Schwikowski B and Ideker T: Cytoscape: A software environment for integrated models of biomolecular interaction networks. Genome Res 13: 2498-2504, 2003.

23. Chen J, Zhao KN and Liu GB: Estrogen-induced cholestasis: Pathogenesis and therapeuticimplications. Hepatogastroenterology 60: 1289-1296, 2013.

24. Glantz A, Marschall HU and Mattsson LA: Intrahepatic cholestasis of pregnancy: Relationships between bile acid levels and fetal complication rates. Hepatology 40: 467-474, 2004.

25. Lammert F, Marschall HU, Glantz A and Matern S: Intrahepatic cholestasis of pregnancy: Molecular pathogenesis, diagnosis and management. J Hepatol 33: 1012-1021, 2000.

26. Xu Y, Yang X, Wang Z, Li M, Ning Y, Chen S, Yin L and Li X Estrogen sulfotransferase (SULT1E1) regulates inflammatory response and lipid metabolism of human endothelial cells via PPARgamma. Mol Cell Endocrinol 369: 140-149, 2013.

27. Liu X, Xue R, Yang C, Gu J, Chen S and Zhang S: Cholestasis-induced bile acid elevates estrogen level via farnesoid $\mathrm{X}$ receptor-mediated suppression of the estrogen sulfotransferase SULT1E1. J Biol Chem 293: 12759-12769, 2018.

28. Yalcin EB, More V, Neira KL, Lu ZJ, Cherrington NJ, Slitt AL and King RS. Downregulation of sulfotransferase expression and activity in diseased human livers. Drug Metab Dispos 41: 1642-1650, 2013.

29. Li L and Falany CN: Elevated hepatic SULT1E1 activity in mouse models of cystic fibrosis alters the regulation of estrogen responsive proteins. J Cyst Fibros 6: 23-30, 2007.

30. Deo AK and Bandiera SM: Identification of human hepatic cytochrome p450 enzymes involved in the biotransformation of cholic and chenodeoxycholic acid. Drug Metab Dispos 36: 1983-1991, 2008

31. Saini SP, Sonoda J, Xu L, Toma D, Uppal H, Mu Y, Ren S, Moore DD, Evans RM and Xie W: A novel constitutive androstane receptor-mediated and CYP3A-independent pathway of bile acid detoxification. Mol Pharmacol 65: 292-300, 2004

32. Li T and Chiang JY: Rifampicin induction of CYP3A4 requires pregnane $X$ receptor cross talk with hepatocyte nuclear factor 4alpha and coactivators, and suppression of small heterodimer partner gene expression. Drug Metab Dispos 34: 756-764, 2006.

33. Honda A, Ikegami T, Nakamuta M, Miyazaki T, Iwamoto J, Hirayama T, Saito Y, Takikawa H, Imawari M and Matsuzaki Y: Anticholestatic effects of bezafibrate in patients with primary biliary cirrhosis treated with ursodeoxycholic acid. Hepatology 57: 1931-1941, 2013.
34. Back P: Therapeutic use of phenobarbital in intrahepatic cholestasis. Inductions in bile acid metabolism. Pharmacol Ther 33: 153-155, 1987.

35. Zhao X, Sheng L, Wang L, Hong J, Yu X, Sang X, Sun Q, Ze Y and Hong F: Mechanisms of nanosized titanium dioxide-induced testicular oxidative stress and apoptosis in male mice. Part Fibre Toxicol 11: 47, 2014

36. Räisänen SR, Lehenkari P, Tasanen M, Rahkila P, Harkonen PL and Väänänen HK: Carbonic anhydrase III protects cells from hydrogen peroxide-induced apoptosis. FASEB J 13: 513-522, 1999.

37. Miethke AG, Zhang W, Simmons J, Taylor AE, Shi T, Shanmukhappa SK, Karns R, White S, Jegga AG, Lages CS, et al: Pharmacological inhibition of apical sodium-dependent bile acid transporter changes bile composition and blocks progression of sclerosing cholangitis in multidrug resistance 2 knockout mice. Hepatology 63: 512-523, 2016.

38. Parkkila S, Halsted $\mathrm{CH}$, Villanueva JA, Väänänen $H K$ and Niemelä O: Expression of testosterone-dependent enzyme, carbonic anhydrase III, and oxidative stress in experimental alcoholic liver disease. Dig Dis Sci 44: 2205-2213, 1999.

39. Zimmerman UJ, Wang P, Zhang X, Bogdanovich S and Forster R: Anti-oxidative response of carbonic anhydrase III in skeletal muscle. IUBMB Life 56: 343-347, 2004.

40. Cabiscol E and Levine RL: Carbonic anhydrase III. Oxidative modification in vivo and loss of phosphatase activity during aging. J Biol Chem 270: 14742-14747, 1995.

41. Hong F and Yang S: Ischemic preconditioning decreased leukotriene $\mathrm{C} 4$ formation by depressing leukotriene $\mathrm{C} 4$ synthase expression and activity during hepatic I/R injury in rats. J Surg Res 178: 1015-1021, 2012

42. Martínez-Clemente M,FerréN,González-Périz A,López-ParraM, Horrillo R, Titos E, Morán-Salvador E, Miquel R, Arroyo V, Funk CD and Clària J: 5-lipoxygenase deficiency reduces hepatic inflammation and tumor necrosis factor $\alpha$-induced hepatocyte damage in hyperlipidemia-prone ApoE-null mice. Hepatology 51: 817-827, 2010.

43. Li SQ, Zhu S, Wan XD, Xu ZS and Ma Z: Neutralization of ADAM8 ameliorates liver injury and accelerates liver repair in carbon tetrachloride-induced acute liver injury. J Toxicol Sci 39: 339-351, 2014.

44. Higuchi Y, Yasui A, Matsuura K and Yamamoto S: CD156 transgenic mice. Different responses between inflammatory types. Pathobiology 70: 47-54, 2002.

45. Li X, Liu R, Luo L, Yu L, Chen X, Sun L, Wang T, Hylemon PB, Zhou H, Jiang $\mathrm{Z}$ and Zhang L: Role of AMP-activated protein kinase $\alpha 1$ in $17 \alpha$-ethinylestradiol-induced cholestasis in rats. Arch Toxicol 91: 481-494, 2017.

46. Li X, Liu R, Zhang L and Jiang Z: The emerging role of AMP-activated protein kinase in cholestatic liver diseases. Pharmacol Res 125: 105-113, 2017.

47. Moustafa T, Fickert P, Magnes C, Guelly C, Thueringer A, Frank S, Kratky D, Sattler W, Reicher H, Sinner F, et al: Alterations in lipid metabolism mediate inflammation, fibrosis, and proliferation in a mouse model of chronic cholestatic liver injury. Gastroenterology 142: 140-151.e12, 2012.

48. Leuenberger N, Pradervand S and Wahli W: Sumoylated PPARalpha mediates sex-specific gene repression and protects the liver from estrogen-induced toxicity in mice. J Clin Invest 119: 3138-3148, 2009.

49. Li T and Chiang JY: Nuclear receptors in bile acid metabolism. Drug Metab Rev 45: 145-155, 2013.

50. Chen P, Li D, Chen Y, Sun J, Fu K, Guan L, Zhang H, Jiang Y, Li X, Zeng X, et al: p53-mediated regulation of bile acid disposition attenuates cholic acid-induced cholestasis in mice. $\mathrm{Br}$ J Pharmacol 174: 4345-4361, 2017.

51. Yang $\mathrm{H}, \mathrm{Li} \mathrm{TW}, \mathrm{Ko} \mathrm{KS}$, Xia $M$ and Lu SC: Switch from Mnt-Max to Myc-Max induces p53 and cyclin D1 expression and apoptosis during cholestasis in mouse and human hepatocytes. Hepatology 49: 860-870, 2009.

52. Wilkins BJ, Lorent K, Matthews RP and Pack M: p53-mediated biliary defects caused by knockdown of cirhla, the zebrafish homolog of the gene responsible for North American Indian Childhood Cirrhosis. PLoS One 8: e77670, 2013.

53. So J, Khaliq M, Evason K, Ninov N, Martin BL, Stainier D and Shin D: Wnt/ $\beta$-catenin signaling controls intrahepatic biliary network formation in zebrafish by regulating notch activity. Hepatology 67: 2352-2366, 2018

54. Okabe H, Yang J, Sylakowski K, Yovchev M, Miyagawa Y, Nagarajan S, Chikina M, Thompson M, Oertel M, Baba H, et al: Wnt signaling regulates hepatobiliary repair following cholestatic liver injury in mice. Hepatology 64: 1652-1666, 2016. 